\title{
Erratum to: Manoeuvring and Control
}

\section{Erratum to:}

Chapter 3 in: M. Renilson, Submarine Hydrodynamics, https://doi.org/10.1007/978-3-319-79057-2_3

In the original version of the book, the belated corrections from author to update Eqs. (3.105)-(3.110) have to be incorporated in Chap. 3. The erratum chapter and the book have been updated with the changes.

The updated online version of this chapter can be found at https://doi.org/10.1007/978-3-319-79057-2_3 